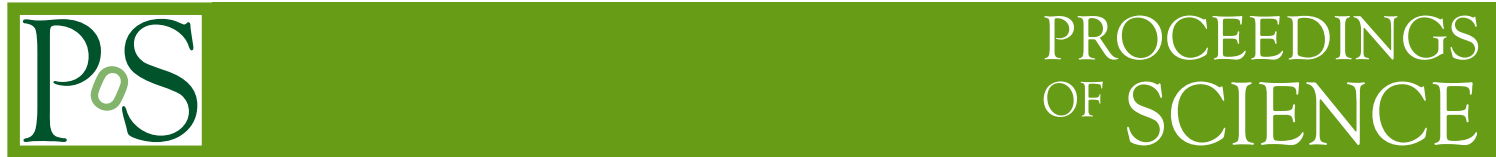

\title{
Inclusive and differential top quark production at the Tevatron
}

Veronica Sorin $* \dagger$

IFAE-Barcelona

E-mail: vsorindifae.es

Latest measurements of top pair production at the Tevatron are summarized. The first combination of precise results from D0 and CDF Collaborations is presented and the individual results discussed. The full Run II data set has been also exploited to look at differential distributions.

Highlights of these recent measurements are reported.

The European Physical Society Conference on High Energy Physics

18-24 July, 2013

Stockholm, Sweden

${ }^{*}$ Speaker.

${ }^{\dagger}$ On behalf of the D0 and CDF Collaborations. 


\section{Introduction}

Since the discovery of the top quark at the Tevatron, the two multipurpose detectors, D0 and $\mathrm{CDF}$, have maintained a comprehensive physics program to study the top quark and its properties. In particular, the measurement of top pair production provides a test of the standard model (SM) predictions and a probe for the presence of possible new physics. At the Tevatron, top quarks are expected, in the SM, to be produced mainly in pairs through strong interactions with approximately $85 \%$ via quark-antiquark annihilation and $15 \%$ from gluon fusion. The top quark decays almost $100 \%$ to a $\mathrm{W}$ boson and a $b$ quark, thus the final states are categorized by the decay of the $\mathrm{W}$ bosons as Lepton+Jets, Dilepton or All-Hadronic.

The latest and most precise measurements of each experiment are reported and results compared with the most recent theoretical predictions. The first combination measurement from the Tevatron is presented and in addition, measurements of differential cross sections are reviewed.

\section{The $t \bar{t}$ cross section in the Lepton+Jets channel}

This channel is populated by events where one W boson decays leptonically and the other hadronically. Leptons in this case corresponds to either identified electrons or muons. Events are selected requiring 3 or more jets, a lepton and missing transverse energy. The CDF collaboration has performed the analysis using two methods, by doing a counting experiment, reducing backgrounds by demanding the presence of jets identified as coming from $b$-hadrons ( $b$-tagging), and by utilizing event kinematic properties to discriminate against backgrounds (topological). Systematic uncertainties are dominated by the $b$-tagging procedure in the first case and the jet energy scale calibration in the other. Uncertainties from the luminosity have been reduced by performing a ratio measurement over the $Z / \gamma^{*} \rightarrow l l$ inclusive cross section , measured in the same corresponding data sample, and thus replaced by its small theoretical uncertainty of $2 \%$ [1]. The most precise result is obtained with the topological method, where a Neural Net output constructed from different event properties is fit using templates built from Monte Carlo simulations for $t \bar{t}$ and the main $\mathrm{W}+\mathrm{jets}$ background. The measured cross sections corresponds to $\sigma(p \bar{p} \rightarrow t \bar{t})=$ $7.82 \pm 0.38$ (stat) \pm 0.41 (syst) pb for a data sample of $4.6 \mathrm{fb}^{-1}$, assuming a mass of $m_{t}=172.5 \mathrm{GeV}$ ${ }^{1}$. The $b$-tagging measurement result is $\sigma(p \bar{p} \rightarrow t \bar{t})=7.32 \pm 0.36$ (stat) \pm 0.61 (syst) pb [⿰]𠃌 ]. Figure 11 shows the jet multiplicity distribution for the $b$-tagged analysis and the result of the fit for the kinematic one.

The D0 collaboration uses also both techniques, $b$-tagging and kinematics, to build a combined analysis. The $t \bar{t}$ candidate sample is divided in regions based on the number of jets and $b$-tags present in the event. This analysis includes the 2 jets sample to derive a correction factor to the $\mathrm{W}+$ heavy flavor contribution determined using Monte Carlo. For regions where background contamination is large, a discriminant is built from different kinematic properties. Different likelihoods are built depending if the region is one where a discriminant was used or just the b-tagging procedure was utilized. All these likelihoods are multiplied and a fit to data is performed to obtain a $t \bar{t}$ cross section value of $\sigma(p \bar{p} \rightarrow t \bar{t})=7.90_{-0.69}^{+0.78}$ (stat + syst) pb for a data sample of $5.3 \mathrm{fb}^{-1}$ [级].

\footnotetext{
${ }^{1}$ Otherwise noted, all results are quoted assuming $m_{t}=172.5 \mathrm{GeV}$
} 

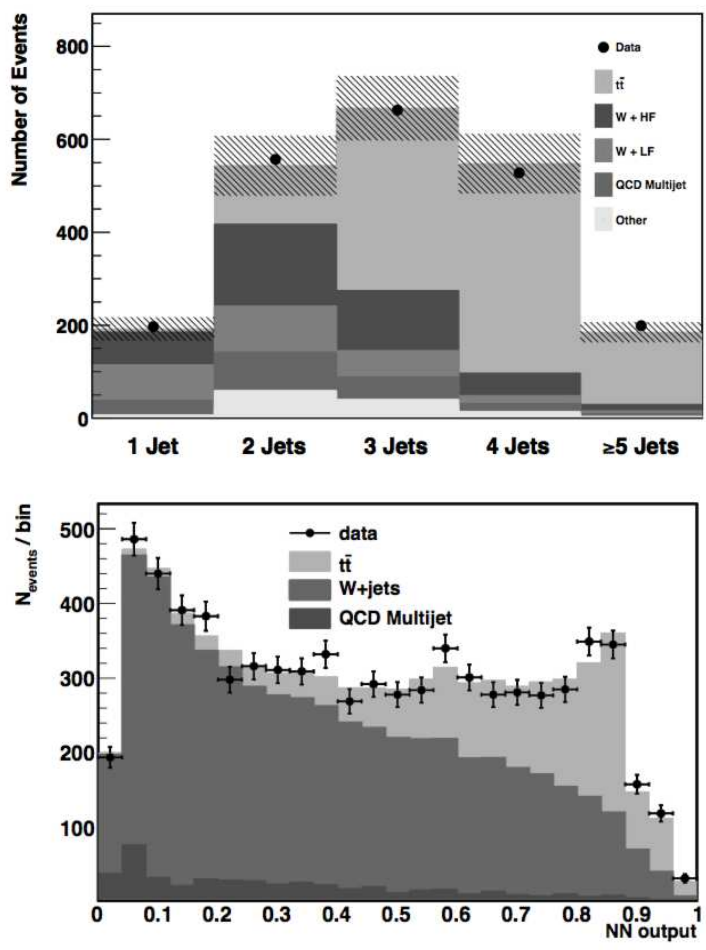

Figure 1: (Top) Jet multiplicity distribution for CDF data $\left(L=4.6 \mathrm{fb}^{-1}\right)$ and predicted background. Hashed lines correspond to the uncertainty on the prediction. (Bottom) Output of the Neural Net for CDF data and SM prediction for $t \bar{t}$ and backgrounds.

\section{The $t \bar{t}$ cross section in the Dilepton channel}

This final state is characterized by the presence of two leptons from the decay of the $\mathrm{W}$ bosons. Backgrounds are dominated by $Z / \gamma^{*}$ production and contamination from $\mathrm{W}+$ jets events where jets are misidentified as leptons. The D0 experiment uses the $e \mu$, ee and $\mu \mu$ final states requiring at least one jet for the $e \mu$ case and at least two for the others. In order to achieve a better separation of the signal, the lowest Neural Net output of the jets $b$-tagging identification procedure is used as discriminant. Figure 2 shows the expected and observed distributions for each region. A simultaneous fit of all channels is performed to obtain a cross section $\sigma(p \bar{p} \rightarrow t \bar{t})=7.36_{-0.79}^{+0.90}$ (stat + syst) pb for a data sample of $5.4 \mathrm{fb}^{-1}[$ [ $]$.

At CDF the dilepton analysis is performed using the full Run II data sample requiring btagging to enhance the signal purity. Control regions are built from the 0 and 1 jet regions while the signal one is constructed requiring 2 jets with at least one of them being b-tagged. In this region, where further requirements of opposite sign leptons and $H_{T}$ (summed transverse energy over all particles) $>200 \mathrm{GeV}$ are applied, the purity is of $91 \%$. Doing a counting experiment a cross section value of $\sigma(p \bar{p} \rightarrow t \bar{t})=7.09 \pm 0.49$ (stat) \pm 0.67 (syst) pb for a data sample of $8.8 \mathrm{fb}^{-1}$ is obtained [5]. 

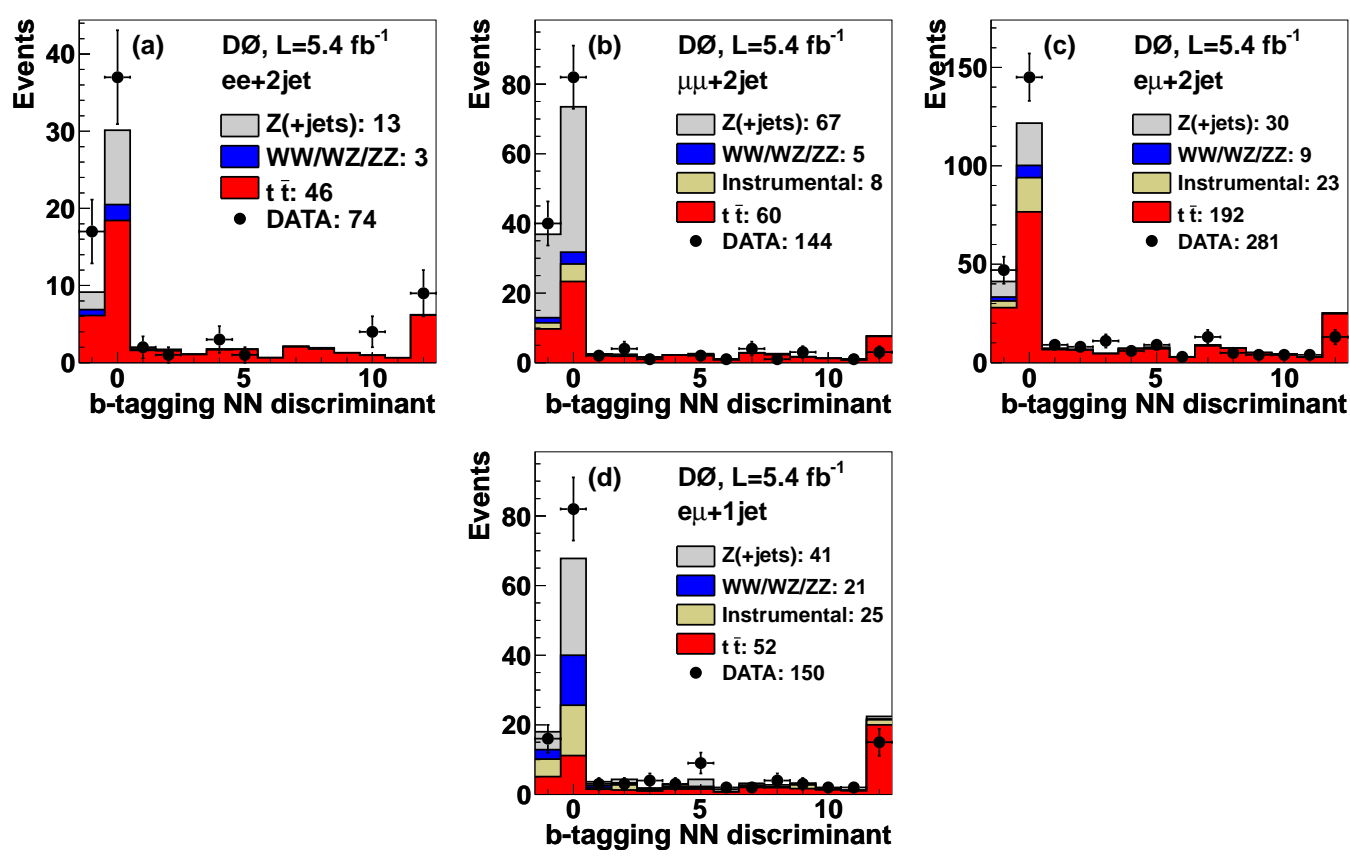

Figure 2: Expected and observed distributions for the b-tagging Neural Net discriminant for each channel: a) $e e+2$ jets, b) $\mu \mu+2$ jets, c) $e \mu+2$ jets and d) $e \mu+1$ jet.

\section{The Tevatron Combination}

Precise measurements for each decay channel have been combined within each experiment and now also combined to produce the first Tevatron result. The combination for CDF includes a measurement from the all hadronic channel. This measurement was performed simultaneously with a top quark mass measurement in samples of 6 to 8 jets and 1 or more b-tag jets. The methodology includes the development of several Neural Nets to suppress backgrounds which is largely composed by multijet production [6]. Using a best linear unbiased estimate (BLUE) method, [7], the CDF combination yields a value of $\sigma(p \bar{p} \rightarrow t \bar{t})=7.63 \pm 0.31$ (stat) \pm 0.36 (syst) \pm 0.15 (lumi) $\mathrm{pb}$. In the case of D0, the Lepton+Jets and Dilepton measurements are combined using the same nuisance-parameter technique as utilized in each individual result obtaining a value of $\sigma(p \bar{p} \rightarrow$ $t \bar{t})=7.56_{-0.56}^{+0.63}($ stat + syst $) \mathrm{pb}[$ [1.

The first Tevatron combination includes the results describe above which are illustrated in Figure 3. All sources of systematic uncertainties have been carefully analyzed and divided in nine classes with similar correlation properties. Using the BLUE method, the combination yields a value of $\sigma(p \bar{p} \rightarrow t \bar{t})=7.60 \pm 0.41 \mathrm{pb}[8]$. This precise result has a similar uncertainty (5.4\%) than that of the theoretical prediction calculated at NNLO+NNLL QCD, $\sigma(p \bar{p} \rightarrow t \bar{t})=7.35_{-0.33}^{+0.28} \mathrm{pb}[9]$.

\section{Differential cross sections}

Differential cross sections have been studied since the top quark discovery. With the full Run II data set more precise measurements have been performed and compared with recent QCD predictions (approximate NNLO calculations) and event generators. In particular, the D0 collaboration 


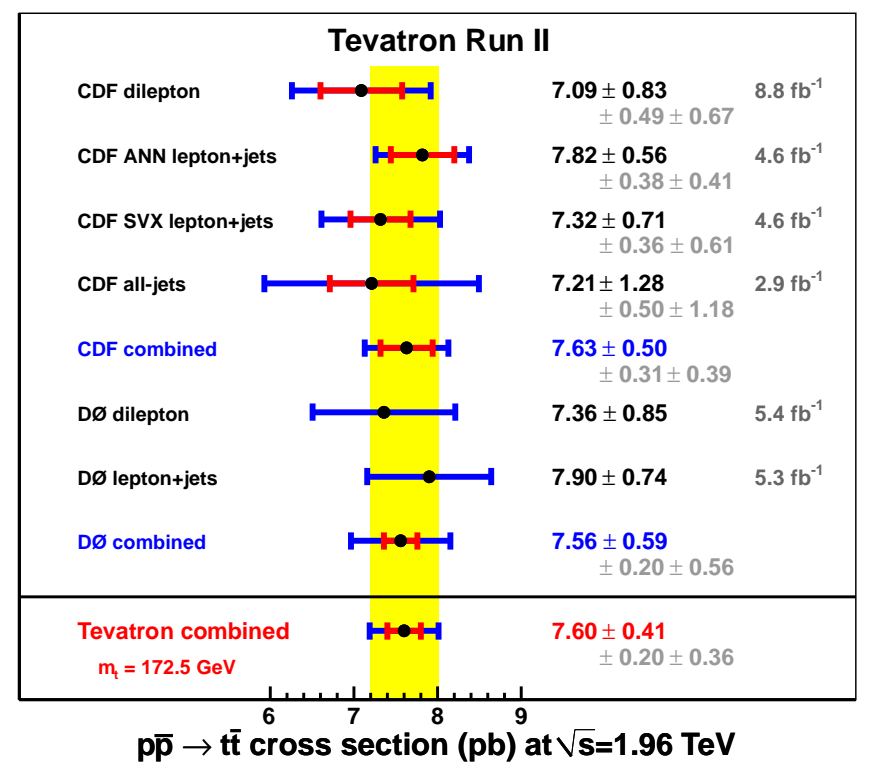

Figure 3: The inputs from the CDF and D0 experiments to the Tevatron combination. Inner bars indicate the statistical uncertainties while the outer bars show the total one.

has explored distributions as a function of transverse momentum, absolute rapidity of the top quark and invariant mass of the $t \bar{t}$ pair in the Lepton+Jets channel. Data is corrected by detector efficiency, acceptance and unfolded to correct for bin migrations. Data is found to be in reasonable agreement with theory within the uncertainties as shown in Figure $\$$ (though ALPGEN is found low in absolute normalization) [10]. Recent CDF studies with the full Run II data set have focused on the differential cross section as function of the top quark production angle. This measurement carries information that could help understanding the large forward-backward asymmetry seen at the Tevatron and it is described in [11] and the related contribution in this proceedings.

\section{Summary}

Latest results from CDF and D0 on inclusive and differential top pair production have been presented. In particular the first Tevatron cross section combination has been discussed and latest differential cross sections using the full Run II data set have been reported. Many more top quark production properties have been investigated at the Tevatron that could not be mentioned in this paper but can be found in [12].

\section{References}

[1] A. Abulencia et al. (CDF Collaboration), J. Phys. G 34, 2457 (2007).

[2] T. Aaltonen et al. (CDF Collaboration), Phys. Rev. Lett. 105, 012001 (2010).

[3] V. M. Abazov et al. (D0 Collaboration), Phys. Rev. D 84, 012008 (2011).

[4] V. M. Abazov et al. (D0 Collaboration), Phys. Lett. B 704, 403 (2011). 

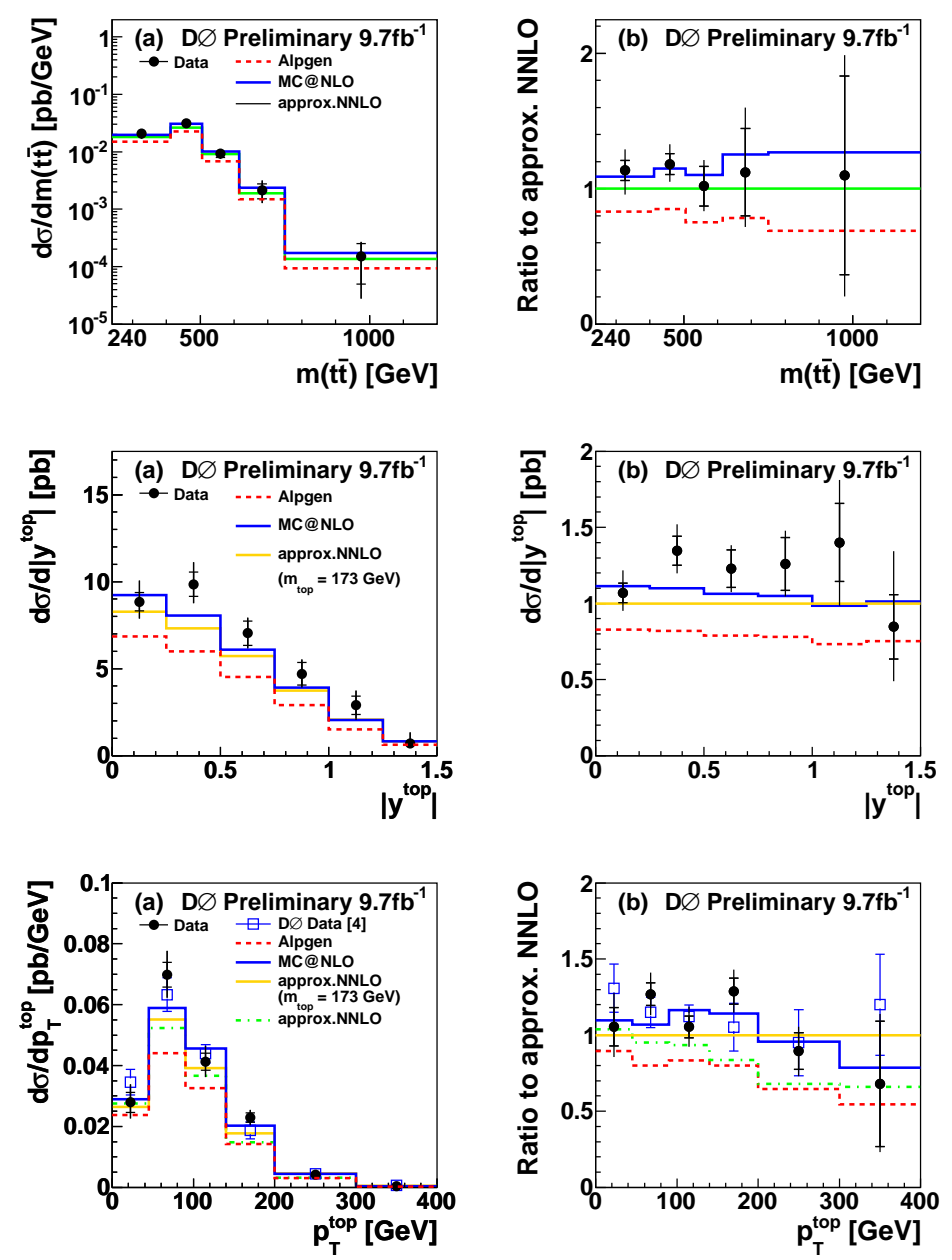

Figure 4: From top to bottom: differential cross sections distributions as a function of the top pair invariant mass, top quark absolute rapidity and transverse momentum compared with several QCD predictions.

[5] T. Aaltonen et al. (CDF Collaboration), [arXiv:1304.7961], submitted to Phys. Rev. Lett..

[6] T. Aaltonen et al. (CDF Collaboration), Phys. Rev. D 81, 052011 (2010)

[7] L. Lyons, D. Gibaut and P. Clifford, Nucl. Instrum. Methods A 270, 110 (1988). L. Lyons, A. Martin and D. Saxon, Phys. Rev. D 41, 3 (1990). A. Valassi, Nucl. Instrum. Methods A 500, 391 (2003).

[8] The CDF and D0 Collaborations, [arXiv:1309.7570], submitted to Phys. Rev. D.

[9] M. Czakon, P. Fiedler and A. Mitov, [arXiv:1303.6254].

[10] The D0 Collaboration, Conference Note 6379

[11] T. Aaltonen et al., The CDF Collaboration, [arXiv:1306.2357], submitted to Phys. Rev. L.

[12] http://www-cdf.fnal.gov/physics/new/top/top.html ;

http://www-d0.fnal.gov/Run2Physics/top/top_public_web_pages/top_public.html ;

Frédéric Déliot, Yvonne Peters and Veronica Sorin, Top Quark Physics at the Tevatron, International Journal of Modern Physics A, 28, 08, 2013 